\title{
A Study to Evaluate Spatial Gait Parameters in Patients of Osteoarthritic Knee
}

\author{
Amanpreet Kaur*, Apoorv Narain**, Jagmohan Singh***, \\ Amandeep Singh**** \\ *MPT 2nd Year Student, **Associate Professor, ***Professor and Principal, Gian Sagar College of \\ Physiotherapy, ****Associate Professor, Gian Sagar Medical College and Hospital, Rajpura, Distt. Patiala, \\ Punjab (INDIA).
}

\begin{abstract}
Osteoarthritis is a slowly evolving articular disease, which appears to originate in the cartilage and affects the underlying bone, soft tissues and synovial fluid. Quantitative gait analysis is an important clinical tool for quantifying normal and pathological patterns of locomotion, and has also been shown to be useful for prescription of treatment as well as in the evaluation of the results of such treatment. The objectives of this study was to evaluate spatial gait parameters variations in patients with osteoarthritic knee as compared to normal healthy individuals. 100 subjects were included in the study, 50 normal healthy subjects and 50 subjects with bilateral knee osteoarthritis with age group of 40-60 years based on the inclusion and exclusion criteria. Before gait analysis, all subjects were given informed consent as advised by the Institutional Ethical Committee. Gait analysis was done for both groups with same protocol. Following gait parameters was measured individually for each subject: Step length [m], Stride length [m], Normalised stride length [\%], Step width [m] and Foot angle [degree]. Paired and unpaired t- test will be used for the analysis of data. The study concluded that there was significant difference is spatial gait parameters in patients with osteoarthritic knee as compared to normal healthy individuals.
\end{abstract}

Keywords: Osteoarthritis, Gait parameters, Spatial gait parameters.

\section{Introduction}

The term osteoarthritis or degenerative arthritis or more appropriately osteoarthrosis is currently used to define an idiopathic slowly progressive disease of synovial joints occurring late in life characterised pathologically by focal degeneration of articular cartilage, subchondral bone thickening, osteophytes and joint deformity (Hvid 1984, Altman 2003). Osteoarthritis (OA) is the most common rheumatological disease which causes physical disability (Buckwalter 2002). Knee osteoarthritis (OA) is a common painful and chronic condition that affects a large proportion of the older population (Access Economics 2007, Woolf AD 2003). At present, musculoskeletal disorders, of which osteoarthritis is the most common, is the fourth leading cause of the economic healthcare burden. Local inflammation in the synovium and the cartilage may contribute to pain and joint damage (Pelletier et al. 2001). The following three joint compartments combine to form the knee: the lateral tibio-femoral compartment, the medial tibio-femoral compartment, and the patella-femoral compartment. Although any of these three compartments may be a source of the pain associated with osteoarthritis, pain emanates most often from the patella-femoral joint (McAlindon et al. 1992), Bone (Felson et al. 2001), synovial inflammation, and a stretched joint capsule filled with fluid are likely to be sources of pain (Hill 2001). Risk factors of osteoarthritis of the knee include older age, female sex, obesity, osteoporosis, occupation, sports activities, previous trauma, muscle weakness or dysfunction, proprioceptive deficit and genetic factors (Bosomworth 2009). The physical disability arising from knee prevents the performance of daily life activities and negatively affects life quality. For most individuals, independent functioning in the community presupposes the ability to walk. It should not be surprising, therefore, that surveys of patients or the families of patients participating in rehabilitation show them to consider walking as a high priority (Nelson et al. 1991). The temporo-spatial parameters are relatively easy to measure, requiring little more than a stopwatch, making them practical for clinical use (Oberg et al. 1993). When walking is impaired, the clinician's role is to determine the nature and severity of the impairment. This is simply a comparison between the patient and a healthy unimpaired individual of similar age and gender. The most common sources of normative data are those collected on "control" groups that are included in studies on pathological gait patterns in patients with osteoarthritic knee for the purpose of comparison. Complex gait analysis presents itself as a possible tool for evaluating the rehabilitation process before and after treatment (Knoll et al. 2004). 


\section{Need of study}

A database of basic gait parameters would be an extremely useful resource for those involved in measuring gait, since virtually all gait analyses include some, if not all, of these basic measurements. Assessment of gait changes at an early stage of osteoarthritic knee will be helpful in planning management for these variations.

\section{Aim of the study}

To evaluate the spatial gait parameters in patients with osteoarhritic knee as compared to normal healthy subjects.

\section{Objectives}

To evaluate spatial gait parameters in patients of osteoarthritic knee.

To evaluate spatial gait parameters in normal subjects.

To compare spatial gait parameters in normal subjects and patients of osteoarthritic knee.

Hypothesis

Null hypothesis: There is no significant difference in various spatial gait parameters of knee osteoarthritis patients and normal subjects. Alternate hypothesis: There is significant difference between various spatial gait parameters of knee osteoarthritis patients and normal subjects.

Study design- Cross-sectional study design comparative in nature.

Sample- 100 subjects.

\section{Inclusion criteria-}

\section{Individuals aged between 40-60 years}

Control Group-Healthy individuals with no associated signs and symptoms related to lower limb musculoskeletal disorders.

Experimental Group- subjects with diagnosis of bilateral knee osteoarthritis based on radiographic evidence and knee pain for more than 6 months as well as at least one of the following: mild swelling, tenderness on palpation, crepitus on motion or stiffness in the morning or after long periods of inactivity.

\section{Exclusion Criteria}

Subjects with radiating pain in the lower limb.

Pregnancy.

Recent history of trauma.

Active inflammation.

Procedure

The 100 subjects were included in the study based on the inclusion and exclusion criteria. Before gait analysis, all subjects gave informed consent as advised by the Ethics Committee. Both groups underwent gait analysis with the same protocol. Following gait parameters will be measured individually for each subject.

Step length $[\mathrm{m}]$, the distance between the ipsilateral and contralateral heel strikes; Stride length $[\mathrm{m}]$, the distance between two consecutive heel strikes of the same foot; Stride length/height [\%], the stride length normalized by subject height; Step width [m], it is the width of the walking base (base of support) is the linear distance between one foot and the opposite foot; Foot angle [degree of toe out or toe in], the angle of foot placement with respect to line of progression. 
Variables

Dependent variables

Step length $[\mathrm{m}]$

Stride length [m]

Normalized Stride length [m]

Step width $[\mathrm{m}]$

Foot angle [degree]

Operational tool

Mechanical gait analyzer.

\section{Data analysis}

Results were analyzed by unpaired t-test between group A and B. Due to the nature of the outcome measures i.e. Step length [m], Stride length [m], Normalized Stride length [m], Step width [m] and Foot angle [degree] unpaired t-test (parametric statistical test) was used to determine the statically significance of the parameters.

\section{Results}

Table 1Descriptive data of Experimental Group A

\begin{tabular}{|l|l|l|}
\hline Dependent Variables & Mean + S.D. & S.E. \\
\hline Step Length ( left) & $0.7546+0.0834$ & 0.011 \\
Step Length ( right) & $0.8022+0.082$ & 0.011 \\
\hline Stride Length ( left) & $1.509+0.161$ & 0.022 \\
Stride Length (right) & $1.574+0.175$ & 0.024 \\
\hline Normalized Stride Length (left) & $0.894+0.119$ & 0.01 \\
Normalized Stride Length (right) & $0.89+0.13$ & 0.018 \\
\hline Step Width & $0.081+0.004$ & 0.00056 \\
\hline Toe- out Angle (left) & $9.34+0.839$ & 0.11 \\
Toe-out Angle (right) & $9.32+0.881$ & 0.12 \\
\hline
\end{tabular}

Table 2Descriptive data of Control Group B

\begin{tabular}{|l|l|l|}
\hline Dependent Variables & Mean + S.D. & S.E. \\
\hline Step Length ( left) & $0.809+0.076$ & 0.0107 \\
Step Length ( right) & $0.865+0.074$ & 0.0104 \\
\hline Stride Length ( left) & $1.62+0.149$ & 0.021 \\
Stride Length (right) & $1.67+0.15$ & 0.021 \\
\hline Normalised Stride Length (left) & $0.964+0.085$ & 0.012 \\
Normalised Stride Length (right) & $0.994+0.086$ & 0.0121 \\
\hline Step Width & $0.074+0.006$ & 0.00084 \\
\hline Toe- out Angle (left) & $6.46+0.85$ & 0.12 \\
Toe-out Angle (right) & $6.42+0.89$ & 0.125 \\
\hline
\end{tabular}

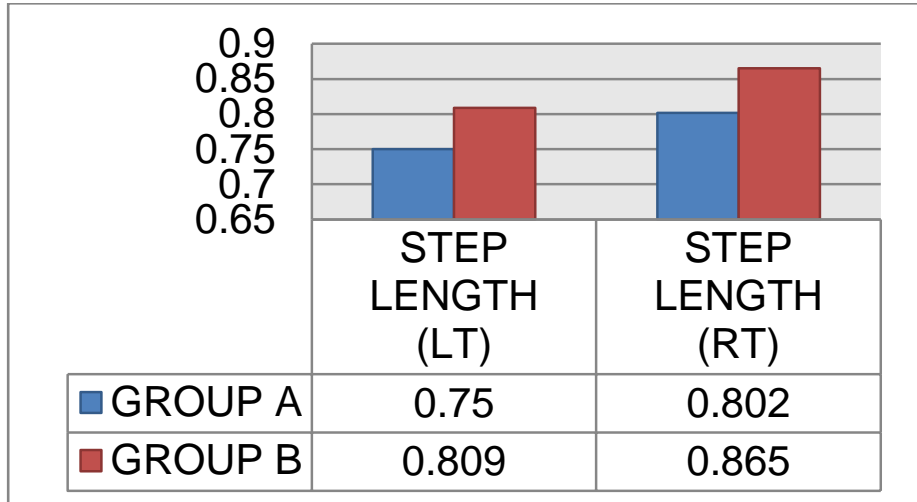

Figure 1: Mean step length variations in group A and B 


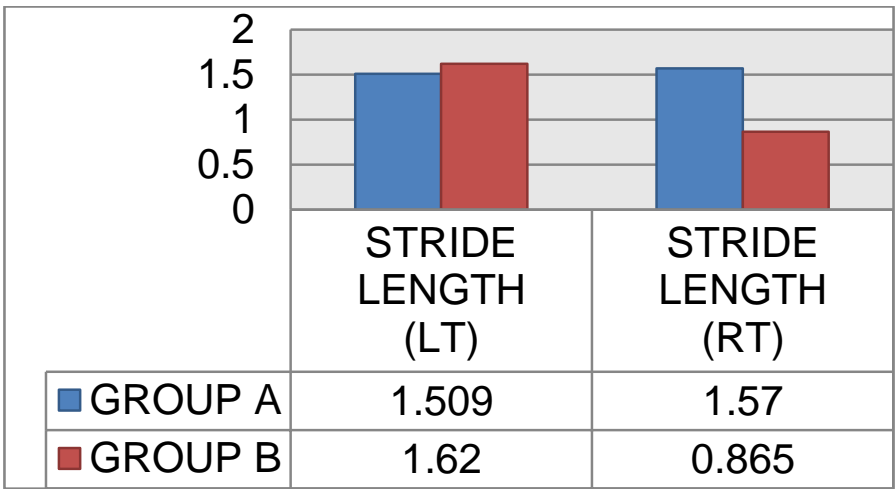

Figure 2: Mean stride length variations in Group A and B

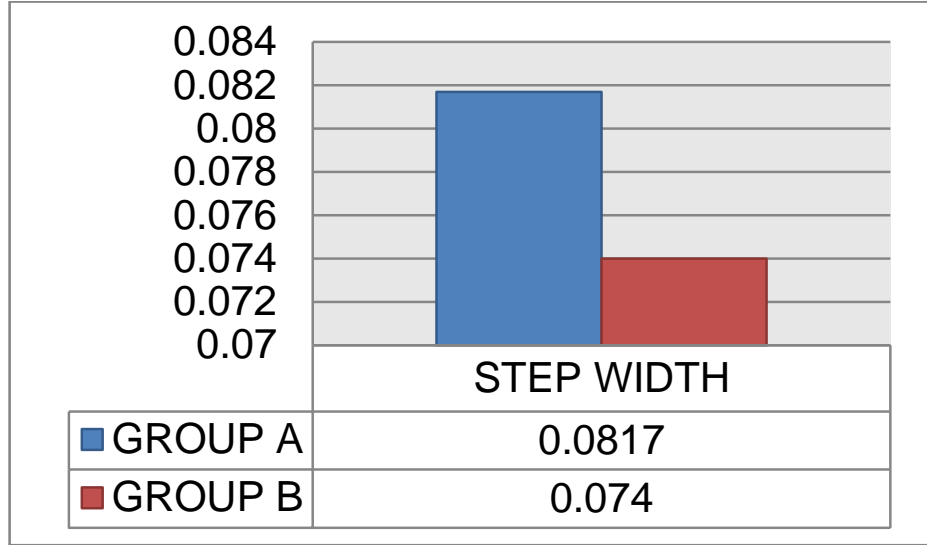

Figure 3: Mean step width variations in Group A and B

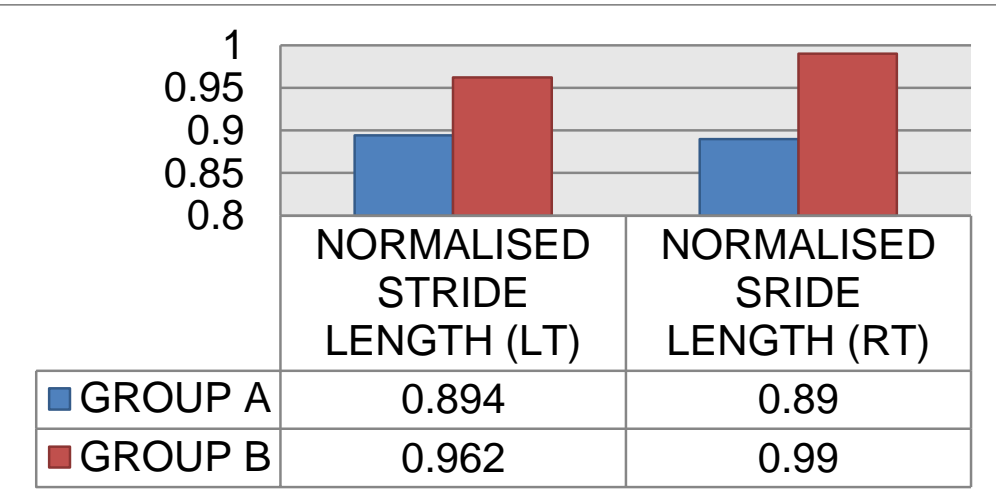

Figure 4: Mean normalized stride length variations in Group A and B

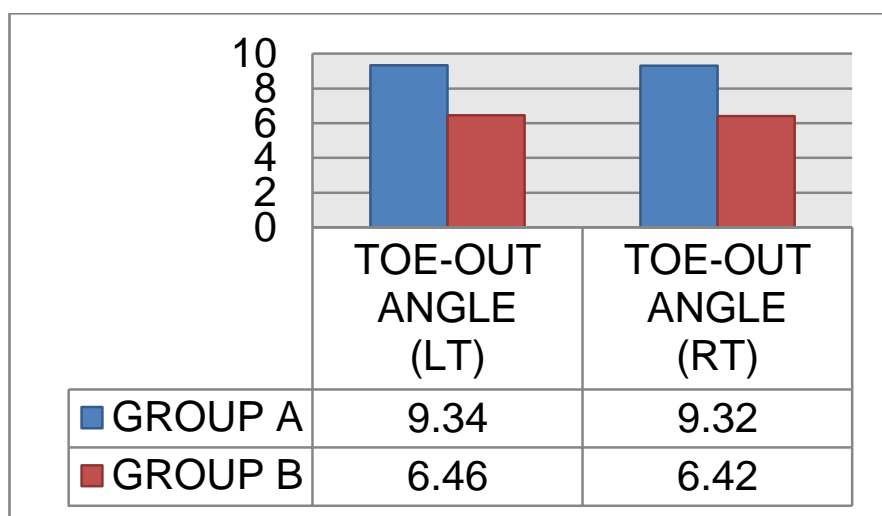

Figure 5: Mean toe-out angle variations in Group A and B 
Table 3Unpaired t-test values using unpaired t-test for comparisons between the groups.

\begin{tabular}{|l|l|l|}
\hline Outcome Measures & $\begin{array}{l}\text { t-calculated (between Group A and } \\
\text { B) }\end{array}$ & Significance \\
\hline Step length (left) & 3.46 & Significant \\
\hline Step length (right) & 4 & Significant \\
\hline Stride length (left) & 3.67 & Significant \\
\hline Stride length (right) & 3.04 & Significant \\
\hline Step width & 6.69 & Significant \\
\hline Normalized stride length (left) & 3.34 & Significant \\
\hline Normalized stride length (right) & 2.38 & Significant \\
\hline Toe-out angle (left) & 16.84 & Significant \\
\hline Toe-out angle (right) & 16.14 & Significant \\
\hline
\end{tabular}

Tabled value at $5 \%$ level of confidence at 48 degree of freedom is 1.660551 .

\section{Discussion}

Osteoarthritis of the knee joint, commonest among all the joints (Mahajan, Verma and Tandon 2005) is likely to become the fourth most important global cause of disability in women and eighth most important in men (Kosuwon et al. 2012). The objective of the present study was to compare the gait patterns of patients with bilateral osteoarthritis of the knee joint to the gait patterns of healthy control subjects. Walking is a common functional activity of daily living. This study provides meaningful information on gait adaptations used by patients with osteoarthritis of the knee joint compared to healthy subjects. Assessment of gait changes will be helpful in the predicting occurrence of compensatory mechanisms during the course of diseases. In our study, the step length, stride length, normalized stride length, step width and toe out angle in patients with knee osteoarthritis changed significantly compared to those of the healthy Subjects. These findings are similar to those found in previous studies (Al- Zahrani and Bakheit, 2002; Andriacchi et al., 1982; Baliunas et al., 2002; Börjesson et al., 2005; Gök et al., 2002; Hurwitz et al., 2000; Kaufmann et al., 2001; Mattson et al., 1990; McGibbon and Krebs, 2002; Messier et al., 1992). According to Hurwitz et al. (2000), the different spatialtemporal parameters were part of the adaptive mechanism to reduce pain, which is similar to the findings of McGibbon and Krebs (2002). The pelvis adaptation enabled the patient to minimize the shorter step length resulting from a reduced motion range of the knee joint and to reduce the pain (Hurwitz et al., 2000). Assessment of gait changes at an early stage of knee osteoarthritis should be helpful in distinguishing between abnormalities causing progression of the disease and occurring as part of the compensatory mechanism during the course of the disease. Spatiotemporal parameters and knee joint angles are considered to be important parameters of gait in knee OA. These may be good predictors of disability (Györy et al. 1976). In our study, spatial parameters were reduced in patients with OA. Similarly, Györy et al. (1976) found reduction in velocity, cadence and stride length in their study. They confirrmed the relationship between these parameters and disability. According to Andriacchi et al. (1982), reduced walking speed and stride length were part of the adaptive mechanism to reduce pain by decreasing knee moments. These adaptive changes occurred relatively early in the course of the disease. However, their clinical significance was not clear since we did not assess the severity of disability and pain, or their relationship with mechanical changes. According to Prodromos et al. (1985), a shorter than normal stride length suggest an unloading mechanism during gait which appears to be beneficial in patients with medial OA of the knee joint which could be a protective mechanism against further progression of the disease by resisting the external adductor moment. Hence, either an increase or decrease in this variable would be a prognostic or etiological factor useful in clinical practice, if shown in future longitudinal studies.

\section{References:}

[1]. Access Economics 2007, 'Painful realities: the economic impact of arthritis in Australia', Arthritis Australia, vol. 21, pp. 23-29.

[2]. Altman, RD, Hochberg, M, Murphy, WA, Wolfe, F and Lequesne, M 1995, 'Atlas of individual radiographic features in osteoarthritis', Osteoarthritis Cartilage, vol. 3, pp. 3-70.

[3]. Al-Zahrani, KS and Bakheit, AM 2002, 'A study of gait characteristic of patients with chronic osteoarthritis of the knee', Disabil Rehabil, vol. 24, pp. 275-280.

[4]. Andriacchi, TP, Galante, J, and Fermier, R 1982, 'The influence of total knee replacement design on walking and stair-climbing', J. Bone Joint Surg Am., vol. 64, pp. 1328-1335.

[5]. Baliunas, AJ, Hurwitz, DE, Ryals, AB, Karrar, A, Case, JP, Block, JA and Andriacchi, TP 2002, 'Increased knee joint loads during walking are present in subjects with knee osteoarthritis', Osteoarthritis Cartilage, vol. 10, pp. 573-579. 
[6]. Buckwalter, JA, Saltzman, C and Brown, T 2004, 'The impact of osteoarthritis: implications for research', Clin Orthop Relat Res, pp. 6-15.

[7]. Felson DT, Niu, J, Guermazi, A, Sack, B and Aliabadi P 2011, 'Defining radiographic incidence and progression of knee osteoarthritis: suggested modifications of the Kellgren and Lawrence scale', Ann Rheum Dis, vol. 70, pp. 1884-6.

[8]. Gyory, AN, Chao, EYS and Stauffer RN 1976, 'Functional evaluation of normal and pathologic knees during gait', Arch Phys Med Rehabil, vol. 57, pp. 571-7.

[9]. Hill, CL, Gale, DR and Chaisson CE 2001, 'Knee effusions, popliteal cysts, and syno- vial thickening: association with knee pain in osteoarthritis', J Rheumatol, vol. 28, pp. 1330-7.

[10]. Hurwitz, DE, Ryals, AR, Block, JA, Sharma, L, Schnitzer, TJ and Andriacchi, TP 2000, 'Knee pain and joint loading in subjects with osteoarthritis of the knee', J Orthop Res, vol. 18, pp. 572-579.

[11]. Hvid, I and Nielsen, S 2001, 'Total condylar knee arthroplasty: prosthetic component positioning and radiolucent lines', Acta Orthop Scand, vol. 55, pp. 160-5.

[12]. Kaufman, KR, Hughes, C, Morrey, BF and Morrey, M 2001, 'Gait characteristics of patients with knee osteoarthritis', J Biomech, vol. 34, no. 7, pp. 907-915.

[13]. Knoll, Zs, Kocsis, L and Kiss, RM 2004, 'Gait patterns before and after anterior cruciate ligament reconstruction', Knee Surgery Sports Traumatology Arthroscopy, vol. 12, pp. 7-13.

[14]. Kosuwon, W, Sirichatiwapee, W, Visanuyotin, T, Jeeravipoolvarn, P and Laupattarakasem, W 2012, 'Determination of Cartilage Volume Using MRI in Patients with Knee Osteoarthritis: Efficacy Study of 25 Milligrams of Sodium Hyaluronate (2.5 M1) Versus Placebo', Clinical and Experimental Pharmacology, vol. 2, no. 2 .

[15]. Mahajan, A, Verma, S and Tandon, V 2005, 'Osteoarthritis', Journal of Associations of Physicians of India, vol, 53, pp, 634-641.

[16]. McAlindon, T, Snow, S, Cooper, C and Dieppe, PA 1992, 'Radiographic patterns of osteoarthritis of the knee joint in the community: the importance of the patellofemoral joint', Ann Rheum Dis, vol. 51, pp. 844-849.

[17]. McGibbon, CA and Krebs, DE 2002, 'Compensatory gait mechanics in patients with unilateral knee arthritis', J Rheumatol., vol. 29, pp. 2410-2419.

[18]. Messier, SP, Glasser, JL, Ettinger, WH, Hassan, BS, Doherty, SA, Mockett, S, Beynnon, BD and Renstrom, PA 2002, 'Declines in strength and balance in older adults with chronic knee pain: a 30-month longitudinal, observational study', Arthritis Rheum, vol. 47, pp.141-148.

[19]. Oberg, T, Karsznia, A and Oberg, K 1993, 'Basic gait parameters: reference data for normal subjects, 10-79 years of age', J Rehabil Res Dev, vol. 30, no. 2, pp. 210-23.

[20]. Pelletier, JP, Martel-Pelletier, J and Abramson, SB 2001, 'Osteoarthritis, an inflammatory disease: potential implication for the selection of new therapeutic targets', Arthritis Rheum, vol. 44, pp. 1237-47. 